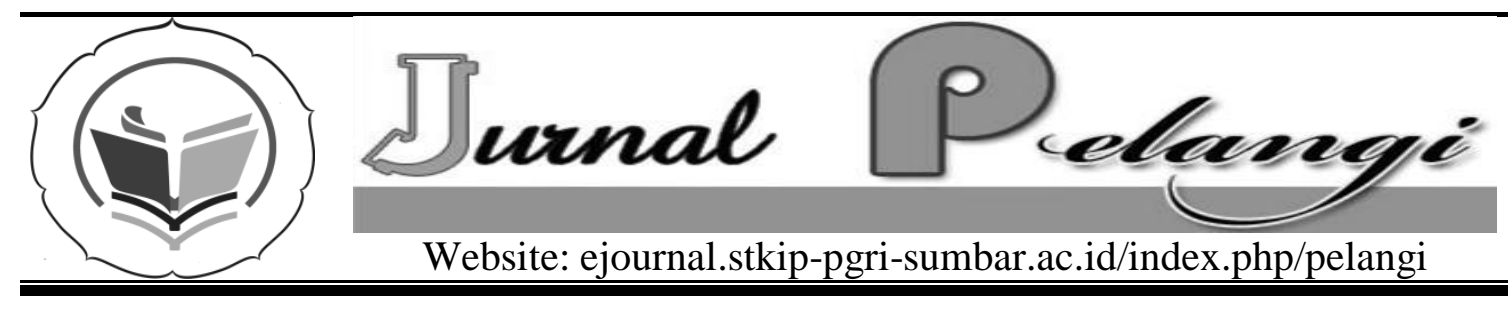

\title{
METODE GARIS SINGGUNG DALAM MENENTUKAN HAMPIRAN INTEGRAL TENTU SUATU FUNGSI PADA SELANG TERTUTUP [ , ]
}

\author{
Zulfaneti dan Rahimullaily* \\ STKIP PGRI Sumatera Barat
}

\section{INFO ARTIKEL}

Diterima:

Direview:

Disetujui:

Keyword:

Riemann integral, approximate method, sum of Riemann, trapezoid method, tangent line method

\section{Abstrak}

There is a value of Riemann integral from a function that cannot be calculated. In this case, approximate method can be used to interpret the value. One of the approximate method of integral Riemann is tangent line method that is a compound method between the sum of Riemann and trapezoid method. The tangent line method uses the tangent line approach. In this writing, the topic discussed is the tangent line method in deciding the approximacy of Riemann integral of a function

\section{Metode Garis Singgung}

Misal $f$ suatu fungsi yang kontinu pada selang tertutup $I=[a, b]$ dan mempunyai turunan di $I . \quad P$ adalah suatu partisi $I$ memakai titik - titik $x_{0}=a<x_{1}<x_{2}<\ldots<x_{n-1}<x_{n}=$ b. Untuk setiap $i=1,2, \ldots, n$, misalkan $\xi_{i} \in I_{i}=\left[x_{i-1}, x_{i}\right], \Delta x_{i}=x_{i}-x_{i-1}$, dan panjang $P$ ditulis $\|P\|$ didefinisikan $\|P\|=$ maks $\Delta x_{i}$. Suatu persamaan garis singgung dari $y=f(x)$ yang melalui titik $\left(\xi_{i}, f\left(\dot{\xi}_{i}\right)\right)$ adalah

$$
g(x)=y=f\left(\xi_{i}\right)+f^{\prime}\left(\xi_{i}\right)\left(x-\xi_{i}\right)
$$

Titik potong garis $g$ dengan garis $x=x_{i-1}$ dan $x=x_{i}$ adalah $\left(x_{i-1}, f\left(\xi_{i}\right)+f^{\prime}\left(\xi_{i}\right)\left(x_{i-1}-\xi_{i}\right)\right) \operatorname{dan}\left(x_{i}, f\left(\xi_{i}\right)+f^{\prime}\left(\xi_{i}\right)\left(x_{i}-\xi_{i}\right)\right)$.

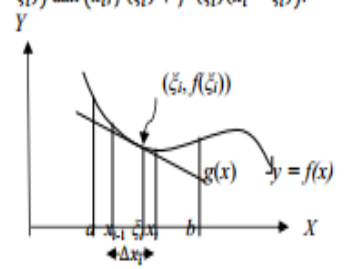

Gambar 1. Hampiran integral tentu dengan metode garis singgung
Berdasarkan Gambar 1, daerah yang dibatasi oleh garis $y=\mathrm{g}(x)=f\left(\xi_{i}\right)+f^{\prime}\left(\xi_{i}\right)\left(x-\xi_{i}\right), x=x_{i-1}, x=x_{i}$ dan $y=0$ berbentuk trapesium yang luasnya

$$
L_{f:\left[x_{i-1}, x_{i}\right]}=\frac{1}{2}\left(f\left(\xi_{i}\right)+f^{\prime}\left(\xi_{i}\right)\left(x_{i-1}-\xi_{i}\right)+f\left(\xi_{i}\right)+f^{\prime}\left(\xi_{i}\right)\left(x_{i-1}-\xi_{i}\right)\right) \Delta x_{i}
$$

$$
=\left(f\left(\xi_{i}\right)+\frac{1}{2} f^{\prime}\left(\xi_{i}\right)\left(x_{i-1}+x_{i-1}-2 \xi_{i}\right)\right) \Delta x_{i}
$$

Jumlah luas $L_{f:\left[x_{i=1}, x_{i}\right]}$ untuk semua $i=1,2, \ldots, n$ adalah

$$
L_{f:[a, b]}=\sum_{i=1}^{n} L_{f:\left[x_{i-1}, x_{i}\right]}=\sum_{i=1}^{n}\left(f\left(\xi_{i}\right)+\frac{1}{2} f^{\prime}\left(\xi_{i}\right)\left(x_{i-1}+x_{i}-2 \xi_{i}\right)\right) \Delta x_{i}
$$

Jika $L_{f:[a, b]}$ mempunyai limit untuk $n$ menuju tak terhingga (sama artinya dengan $\|P\|$ menuju 0 ), maka fungsi $f$ terintegralkan dalam metode garis singgung pada $l$.

Definisi 1

Misalkan fungsi f kontinu pada selang tertutup $I=[a, b]$, mempunyai turunan di $I$, dan $a=x_{0}<x_{1}<x_{2}<\ldots<x_{n-1}<x_{n}=b$ adalah suatu partisi $I$, tulis $P$. Untuk setiap $i=1,2, \ldots, n$, misalkan $\xi_{i} \in I_{i}=\left[x_{i-1}, x_{i}\right], \Delta x_{i}=x_{i}-x_{i-1}$, dan panjang $P$ ditulis $\|P\|$ didefinisikan $\|P\|=$ maks $\Delta x_{i}$. Jiko

$$
\sum_{i=1}^{n}\left(f\left(\xi_{i}\right)+\frac{1}{2} f^{\prime}\left(\xi_{i}\right)\left(x_{i-1}+x_{i}-2 \xi_{i}\right)\right) \Delta x_{i} \quad \ldots 1
$$


memiliki limit $\mathrm{n}$ menuju tak terhingga (sama artinya dengan \|\| menuju 0) maka limit tersebut disebut integral garis singgung dan $\mathrm{f}$ terintegralkan dalam metode garis singgung $\mathrm{f}$ pada I, ditulis

$$
\operatorname{cs} \int[a, b] f=\lim _{n \rightarrow \infty} \sum_{i=1}^{n}\left(f\left(\xi_{i}\right)+\frac{1}{2} f^{\prime}\left(\xi_{i}\right)\left(x_{i-1}+x_{i}-2 \xi_{i}\right)\right) \Delta x_{i} \ldots 2
$$

Definisi 2

Fungsif yang terdefinisi pada satu titik a maka cs $\int_{[a, a]} f=0$.

Misalkan cos $\int_{[a, b]} f$ maka as $\int_{[b, a]} f=\boldsymbol{- C S}_{[a, b]} f$.

Dalam hal ini ${ }_{c s} \int_{[b, a]} f$ menyatakan batas integrasinya dari $b$ ke $a$, bukan integral pada $[b, a]$.

\section{Teorema 3}

Misalkan as $\int_{[a, b] f_{1}}$ dan ${ }_{\text {CS }} \int_{[a, b]} f_{2}$ dan misalkan $C_{1}$ dan $C_{2}$ sebarang konstanta, maka:
(i) $\quad$ cs $\int_{[a, b]} C_{1} f_{1} d a n \quad$ cs $\int_{[a p]} C_{2} f_{2}$
(ii) $\quad$ CS $\int_{[a, b]}\left(C_{1} f_{1}+C_{2} f_{2}\right)=C_{1 G S} \int_{[a, b]} f_{1}+C_{2 G S} \int_{[a, b]} f_{2}$

Bukti:

(i) Menurut Definisi 1, maka

$$
\text { c.s } \int[a, b] f_{1}=\lim _{n \rightarrow \infty} \sum_{i=1}^{n}\left(f_{1}\left(\xi_{i}\right)+\frac{1}{2} f_{1}^{\prime}\left(\xi_{i}\right)\left(x_{i-1}+x_{i}-2 \xi_{i}\right)\right) \Delta x_{i}
$$

Jika kedua ruas dikalikan $\mathcal{C}_{1}$, maka

$$
\begin{aligned}
& \qquad \begin{aligned}
C_{1} c s \int_{[a, b]} f_{1} & =C_{1}\left(\lim _{n \rightarrow \infty} \sum_{i=1}^{n}\left(f_{1}\left(\xi_{i}\right)+\frac{1}{2} f_{1}^{\prime}\left(\xi_{i}\right)\left(x_{i-1}+x_{i}-2 \xi_{i}\right)\right) \Delta x_{i}\right) \\
& =\lim _{n \rightarrow \infty} \sum_{i=1}^{n}\left(C_{1} f_{1}\left(\xi_{i}\right)+\frac{1}{2} C_{1} f_{1}^{\prime}\left(\xi_{i}\right)\left(x_{i-1}+x_{i}-2 \xi_{i}\right)\right) \Delta x_{i}
\end{aligned} \\
& =\int_{[a, b]} C_{1} f_{1}
\end{aligned}
$$

(ii) Menurut Definisi 1, maka

cs $\int_{[a, b]}\left(C_{1} f_{1}+C_{2} f_{2}\right)$

$=\lim _{n \rightarrow \infty} \sum_{i=1}^{n}\left(c_{1} f_{1}\left(\xi_{i}\right)+C_{2} f_{2}\left(\xi_{i}\right)+\frac{1}{2}\left(C_{1} f_{1}^{\prime}\left(\xi_{i}\right)+C_{2} f_{2}^{\prime}\left(\xi_{i}\right)\right)\left(x_{i-1}+x_{i}-2 \xi_{i}\right)\right) \Delta x_{i}$

$=C_{1}\left(\lim _{n \rightarrow \infty} \sum_{i=1}^{n}\left(f_{1}\left(\xi_{i}\right)+\frac{1}{2} f_{1}^{\prime}\left(\xi_{i}\right)\left(x_{i-1}+x_{i}-2 \xi_{i}\right)\right) \Delta x_{i}\right)$

$$
+C_{2}\left(\lim _{n \rightarrow \infty} \sum_{i=1}^{n}\left(f_{2}\left(\xi_{i}\right)+\frac{1}{2} f_{2}^{\prime}\left(\xi_{i}\right)\left(x_{i-1}+x_{i}-2 \xi_{i}\right)\right) \Delta x_{i}\right)
$$

$=C_{1_{G S}} \int_{[a, b]} f_{1}+C_{2_{C S}} \int_{[a, b]} f_{2}$ (i) Misalkan $f$ sebarang fungsi kontinu pada selang tertutup $I=[a, b]$ dan mempunyai turunan di $l$, dan misalkan $\xi_{i}=\frac{1}{2}\left(x_{i-1}+x_{i}\right)$ dengan $i=1,2$, ..., $n$ maka

$$
\text { CS } \int_{[a, b]} f={ }_{R} \int_{[a, b]} f
$$

Bukti:

Misalkan $f$ sebarang fungsi kontinu pada selang tertutup $I=[a, b]$ dan mempunyai turunan di $I$, dan misalkan $\xi_{i}=\frac{1}{2}\left(x_{i-1}+x_{i}\right)$ dengan $i=1,2$, ...,n maka diperoleh $x_{i-1}+x_{i}-2 \xi_{l}=0$.

Menurut Definisi 1 maka

$$
\begin{aligned}
G S \int_{[a, b]} f & =\lim _{n \rightarrow \infty} \sum_{i=1}^{n}\left(f\left(\xi_{i}\right)+\frac{1}{2} f^{\prime}\left(\xi_{i}\right)\left(x_{i-1}+x_{i}-2 \xi_{i}\right)\right) \Delta x_{i} \\
& =\lim _{n \rightarrow \infty} \sum_{i=1}^{n}\left(f\left(\xi_{i}\right)+\frac{1}{2} f^{\prime}\left(\xi_{i}\right)(0)\right) \Delta x_{i} \\
& =\lim _{n \rightarrow \infty} \sum_{i=1}^{n} f\left(\xi_{i}\right) \Delta x_{i} \\
& =\int_{R} f
\end{aligned}
$$

(ii) Misalkan $f(x)=t x+s$ dengan $t$ dan $s$ merupakan konstanta dan $\xi_{i}$ sebarang dengan $i=1,2, \ldots, n$, maka

Misalkan $f(x)=t x+s$ dengan $t$ dan $s$ merupakan konstanta, $f(x)$ fungsi polynomial maka $f(x)$ kontinu pada setiap bilangan riil, dan mempunyai turunan yaitu

$$
f^{\prime}(x)=f^{\prime}(t x+s)=f^{\prime}(t x)+f^{\prime}(s)=t f^{\prime}(x)+0=t
$$

untuk semua $x$ di $I=[a, b]$. Misalkan juga $\xi_{i}$ sebarang dengan $i=1,2$, $\ldots, n$, sehingga menurut Definisi 1

$$
\begin{aligned}
\text { as } \int[a, b] & =\lim _{n \rightarrow \infty} \sum_{i=1}^{n}\left(f\left(\xi_{i}\right)+\frac{1}{2} f^{\prime}\left(\xi_{i}\right)\left(x_{i-1}+x_{i}-2 \xi_{i}\right)\right) \Delta x_{i} \\
& =\lim _{n \rightarrow \infty} \sum_{i=1}^{n}\left(\left(t \xi_{i}+s\right)+\frac{1}{2} t\left(x_{i-1}+x_{i}-2 \xi_{i}\right)\right) \Delta x_{i} \\
& =\lim _{n \rightarrow \infty} \sum_{i=1}^{n} \frac{1}{2}\left(\left(t x_{i-1}+s\right)+\left(t x_{i}+s\right)\right) \Delta x_{i}
\end{aligned}
$$

$$
\begin{aligned}
& =\lim _{n \rightarrow \infty} \sum_{i=1}^{n} \frac{1}{2}\left(f\left(x_{i-1}\right)+f\left(x_{i}\right)\right) \Delta x_{i} \\
& ={ }_{T} \int_{[a, b]} f
\end{aligned}
$$

(iii) Misalkan $f$ merupakan fungsi konstan dan misalkan sebuah titik sampel sebarang dari $l_{i}$ yaitu $\xi_{i}$ dengan $i=1,2, \ldots, n$, maka

Bukti :

$$
\text { cs } \int[a, b] f=R \int_{[a, b]} f
$$

Misalkan $f$ merupakan fungsi konstan. Fungsi konstan merupakan fungsi polynomial, maka $f$ kontinu pada setiap bilangan riil akibatnya turunan dari $f$ yaitu $f^{\prime}(x)=0$. Misalkan juga $\xi_{i}$ sebarang dengan $i=1,2, \ldots, n$. Menurut Definisi 1, maka

$$
\text { as } \begin{aligned}
\int[a, b] & =\lim _{n \rightarrow \infty} \sum_{i=1}^{n}\left(f\left(\xi_{i}\right)+\frac{1}{2} f^{\prime}\left(\xi_{i}\right)\left(x_{i-1}+x_{i}-2 \xi_{i}\right)\right) \Delta x_{i} \\
& =\lim _{n \rightarrow \infty} \sum_{i=1}^{n}\left(f\left(\xi_{i}\right)+\frac{1}{2}(0)\left(x_{i-1}+x_{i}-2 \xi_{i}\right)\right) \Delta x_{i} \\
& =\lim _{n \rightarrow \infty} \sum_{i=1}^{n} f\left(\xi_{i}\right) \Delta x_{i} \\
& ={ }_{R} \int_{[a, b]} f
\end{aligned}
$$


Teorema 4

Jika fungsi f terintegralkan ${ }_{G S} \int_{[a, b]}$ maka fungsi f terintegralkan ${ }_{R} \int_{[a, b]} f$ Bukti:

Fungsi $f$ terintegralkan ${ }_{C S} \int_{[a, b]} f$. Menurut Definisi 1

$$
\begin{aligned}
\text { Gs } \int[a, b] f & =\lim _{n \rightarrow \infty} \sum_{i=1}^{n}\left(f\left(\xi_{i}\right)+\frac{1}{2} f^{\prime}\left(\xi_{i}\right)\left(x_{i-1}+x_{i}-2 \xi_{i}\right)\right) \Delta x_{i} \\
& =\lim _{n \rightarrow \infty}\left(\sum_{i=1}^{n} f\left(\xi_{i}\right) \Delta x_{i}+\sum_{i=1}^{n} \frac{1}{2} f^{\prime}\left(\xi_{i}\right)\left(x_{i-1}+x_{i}-2 \xi_{i}\right) \Delta x_{i}\right) \\
& =\lim _{n \rightarrow \infty} \sum_{i=1}^{n} f\left(\xi_{i}\right) \Delta x_{i}+\lim _{n \rightarrow \infty} \sum_{i=1}^{n} \frac{1}{2} f^{\prime}\left(\xi_{i}\right)\left(x_{i-1}+x_{i}-2 \xi_{i}\right) \Delta x_{i}
\end{aligned}
$$

Karena nilai $\lim _{n \rightarrow \infty} \sum_{i=1}^{n} \frac{1}{2} f^{\prime}\left(\xi_{i}\right)\left(x_{i-1}+x_{i}-2 \xi_{i}\right) \Delta x_{i}=0$ maka diperoleh

$$
\begin{aligned}
& \text { CS. } \int_{[a, b]} f=\lim _{n \rightarrow \infty} \sum_{i=1}^{n} f\left(\xi_{i}\right) \Delta x_{i}+0 \\
& =\lim _{n \rightarrow \infty} \sum_{i=1}^{n} f\left(\xi_{i}\right) \Delta x_{i} \\
& =\int_{R} \int_{[a, b]} f
\end{aligned}
$$

Pembuktian $\lim _{n \rightarrow \infty} \sum_{i=1}^{n} \frac{1}{2} f^{\prime}\left(\xi_{i}\right)\left(x_{i-1}+x_{i}-2 \xi_{i}\right) \Delta x_{i}=0$

$n \rightarrow \infty$ sama artinya dengan $\|P\| \rightarrow 0$, schingga

$$
\begin{aligned}
& \lim _{n \rightarrow \infty} \sum_{i=1}^{n} \frac{1}{2} f^{\prime}\left(\xi_{i}\right)\left(x_{i-1}+x_{i}-2 \xi_{i}\right) \Delta x_{i}=\lim _{|P| \rightarrow 0} \sum_{i=1}^{n} \frac{1}{2} f^{\prime}\left(\xi_{i}\right)\left(x_{i-1}+x_{i}-2 \xi_{i}\right) \Delta x_{i} \\
& =\lim _{\| P \mid \rightarrow 0}\left(\frac{1}{2} f^{\prime}\left(\xi_{1}\right)\left(x_{0}+x_{1}-2 \xi_{1}\right) \Delta x_{1}+\frac{1}{2} f^{\prime}\left(\xi_{2}\right)\left(x_{1}+x_{2}-2 \xi_{2}\right) \Delta x_{2}+\cdots\right. \\
& \left.\quad \quad+\frac{1}{2} f^{\prime}\left(\xi_{n}\right)\left(x_{n-1}+x_{n}-2 \xi_{n}\right) \Delta x_{n}\right) \\
& =\sum_{i=1}^{n} \lim _{\| P \mid \rightarrow 0} \frac{1}{2} f^{\prime}\left(\xi_{i}\right)\left(x_{i-1}+x_{i}-2 \xi_{i}\right) \Delta x_{i} \\
& =\sum_{i=1}^{n} \frac{1}{2} \lim _{\|P\| \rightarrow 0} f^{\prime}\left(\xi_{i}\right) \cdot \lim _{\| P \mid \rightarrow 0}\left(x_{i-1}+x_{i}-2 \xi_{i}\right) \cdot \lim _{|P| \rightarrow 0} \Delta x_{i}=0
\end{aligned}
$$

Akan dibuktikan $\lim _{\| P \mid \rightarrow 0}\left(x_{i=1}+x_{i}-2 \xi_{i}\right)=0$. Ambil $\varepsilon>0$ dan pilih $\delta=\frac{t}{2}>0$ maka, $0<\|P\|<\delta$ berarti

$$
\begin{aligned}
\left|x_{i-1}+x_{i}-2 \xi_{i}\right| & =\left|x_{i-1}+x_{i}-2 \xi_{i}\right|=\left|x_{i-1}-\xi_{i}+x_{i}-\xi_{i}\right| \\
& \leq\left|x_{i-1}-\xi_{i}\right|+\left|x_{i}-\xi_{i}\right| \\
& \leq\left|x_{i=1}-x_{i}\right|+\left|x_{i}-x_{i-1}\right| \\
& \leq 2\|P\| \\
& \leq 2 \cdot \frac{\varepsilon}{2}=\varepsilon \\
0<\|P\|<\delta & \Rightarrow\left|x_{i=1}+x_{i}-2 \xi_{i}\right|<\varepsilon
\end{aligned}
$$

Teorema 5

Jika fungsi f terintegralkan ${ }_{R} \int_{[a, b]} f$ dan mempunyai turuman pada $[a, b]$, maka fungsif terintegralkan ${ }_{\text {cS }} \int_{[a, b]} f$

Bukti:

Fungsi f terintegralkan ${ }_{R} \int_{[a, b]} f$, maka

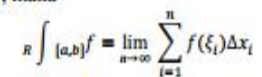

Fungsi $f$ mempunyai turunan pada $[a, b]$. Misalkan $\xi_{i} \in[a, b]$, maka turunan fungsi $f$ ada nilainya pada $\xi_{i}$ (dengan kata lain $f^{\prime}\left(\xi_{i}\right)$ ada). akibatnya $f$ kontinu pada $[a, b]$.

Kedua ruas pada 3 ditambah denga

$$
\lim _{n \rightarrow \infty} \sum_{i=1}^{n} \frac{1}{2} f^{\prime}\left(\xi_{i}\right)\left(x_{i-1}+x_{i}-2 \xi_{i}\right) \Delta x_{i}
$$

maka

$$
\begin{aligned}
& =\int[(\alpha, b])+\lim _{n \rightarrow \infty} \sum_{i=1}^{n} \frac{1}{2} f^{\prime}\left(\xi_{i}\right)\left(x_{i-1}+x_{i}-2 \xi_{i}\right) \Delta x_{i} \\
= & \lim _{n \rightarrow \infty} \sum_{i=1}^{n} f\left(\xi_{i}\right) \Delta x_{i}+\lim _{n \rightarrow \infty} \sum_{i=1}^{n} \frac{1}{2} f^{\prime}\left(\xi_{i}\right)\left(x_{i-1}+x_{i}-2 \xi_{i}\right) \Delta x_{i} \\
= & \lim _{n \rightarrow \infty}\left(\sum_{i=1}^{n} f\left(\xi_{i}\right) \Delta x_{i}+\sum_{i=1}^{n} \frac{1}{2} f^{\prime}\left(\xi_{i}\right)\left(x_{i-1}+x_{i}-2 \xi_{i}\right) \Delta x_{i}\right) \\
= & \lim _{n \rightarrow \infty} \sum_{i=1}^{n}\left(f\left(\xi_{i}\right)+\frac{1}{2} f^{\prime}\left(\xi_{i}\right)\left(x_{i-1}+x_{i}-2 \xi_{i}\right)\right) \Delta x_{i}
\end{aligned}
$$

$$
\begin{aligned}
& \mathbf{=}_{G S} \int_{[a, b]} f \\
& \text { Pada Teorema } 4 \text { telah dibuktikan bahwa } \\
& \lim _{n \rightarrow \infty} \sum_{i=1}^{n} \frac{1}{2} f^{\prime}\left(\xi_{i}\right)\left(x_{i-1}+x_{i}-2 \xi_{i}\right) \Delta x_{i}=0 \\
& \text { sehingga diperoleh } \\
& { }_{R} \int[a, b] f+\lim _{n \rightarrow \infty} \sum_{i=1}^{n} \frac{1}{2} f^{\prime}\left(\xi_{i}\right)\left(x_{i-1}+x_{i}-2 \xi_{i}\right) \Delta x_{i}={ }_{R} \int[a, b] f+0={ }_{C S} \int[a, b] f \\
& \sum_{i=1}^{n}\left(f\left(\xi_{i}\right)+\frac{1}{2} f^{\prime}\left(\xi_{i}\right)\left(x_{i-1}+x_{i}-2 \xi_{i}\right)\right) \Delta x_{i}>\sum_{i=1}^{n} f\left(\xi_{i}\right) \Delta x_{i}
\end{aligned}
$$

dengan kata lain, nilai hampiran integral tentu dari fungsi f menggunakan dengan metode garis singgung lebih besar dibandingkan dengan nilai hampiran jumlah Rieman.

$$
\begin{aligned}
\text { (ii) } j i k a & \xi_{i}>\frac{1}{2}\left(x_{i-1}+x_{i}\right), f^{\prime}\left(\xi_{i}\right)>0 \text { atau } \xi_{i}<\frac{1}{2}\left(x_{i-1}+x_{i}\right), f^{\prime}\left(\xi_{i}\right)<0 \text { untuk setiap } \\
I_{i}, i= & 1,2, \ldots, n \text { maka } \\
& \sum_{i=1}^{n}\left(f\left(\xi_{i}\right)+\frac{1}{2} f^{\prime}\left(\xi_{i}\right)\left(x_{i-1}+x_{i}-2 \xi_{i}\right)\right) \Delta x_{i}<\sum_{i=1}^{n} f\left(\xi_{i}\right) \Delta x_{i}
\end{aligned}
$$

dengan kata lain, nilai hampiran nilai integral tentu dari fungsi f menggunakan hampiran jumlah Riemann besar dibandingkan dengan nilai hampiran dengan metode garis singgung.

Bukti:

$$
\begin{aligned}
& \text { (i) Perhatikan bahwa } \\
& \xi_{i}<\frac{1}{2}\left(x_{i-1}+x_{i}\right) \text { atau } \frac{1}{2}\left(x_{i-1}+x_{i}\right)>\xi_{i} \\
& \text { sehingga } \frac{1}{2}\left(x_{i-1}+x_{i}\right)-\xi_{i}>\xi_{i}-\xi_{i} \text { atau } \frac{1}{2}\left(x_{i-1}+x_{i}-2 \xi_{i}\right)>0 \\
& \text { dan } f^{\prime}\left(\xi_{i}\right)>0 \text { maka } f^{\prime}\left(\xi_{i}\right) \cdot \frac{1}{2}\left(x_{i-1}+x_{i}-2 \xi_{i}\right)>0 \text { sehingga } \\
& \sum_{i=1}^{n} f\left(\xi_{i}\right) \Delta x_{i}+\sum_{i=1}^{n} f^{\prime}\left(\xi_{i}\right) \cdot \frac{1}{2}\left(x_{i-1}+x_{i}-2 \xi_{i}\right) \Delta x_{i}>\sum_{i=1}^{n} f\left(\xi_{i}\right) \Delta x_{i} \\
& \sum_{i=1}^{n}\left(f\left(\xi_{i}\right)+\frac{1}{2} f^{\prime}\left(\xi_{i}\right)\left(x_{i-1}+x_{i}-2 \xi_{i}\right)\right) \Delta x_{i}>\sum_{i=1}^{n} f\left(\xi_{i}\right) \Delta x_{i}
\end{aligned}
$$

(ii) Perhatikan bahwa

$$
\xi_{i}>\frac{1}{2}\left(x_{i-1}+x_{i}\right) \text { atau } \frac{1}{2}\left(x_{i-1}+x_{i}\right)<\xi_{i} \text { atau } \frac{1}{2}\left(x_{i-1}+x_{i}-2 \xi_{i}\right)<0
$$

dan $f^{\prime}\left(\xi_{i}\right)>0$ maka $f^{\prime}\left(\xi_{i}\right) \cdot \frac{1}{2}\left(x_{i-1}+x_{i}-2 \xi_{i}\right)<0$ sehingga

$$
\begin{gathered}
\sum_{i=1}^{n} f\left(\xi_{i}\right) \Delta x_{i}+\sum_{i=1}^{n} f^{\prime}\left(\xi_{i}\right) \cdot \frac{1}{2}\left(x_{i-1}+x_{i}-2 \xi_{i}\right) \Delta x_{i}<\sum_{i=1}^{n} f\left(\xi_{i}\right) \Delta x_{i} \\
\sum_{i=1}^{n}\left(f\left(\xi_{i}\right)+\frac{1}{2} f^{\prime}\left(\xi_{i}\right)\left(x_{i-1}+x_{i}-2 \xi_{i}\right)\right) \Delta x_{i}<\sum_{i=1}^{n} f\left(\xi_{i}\right) \Delta x_{i}
\end{gathered}
$$

Contoh Perhitungan Hampiran Integral Tentu dengan Metode Garis Singgung Telah diketahui bahwa terdapat 3 jenis 
fungsi yang dapat terintegralkan Riemann pada selang tertutup $[a, b]$ dan menurut Teorema 5 maka fungsi tersebut harus memiliki turunan pada $[\mathrm{a}, \mathrm{b}]$ untuk dapat diintegralkan dengan metode garis singgung. Pada bagian ini akan dibahas contoh dari 3 jenis fungsi tersebut dan satu contoh yang sulit diselesaikan dengan teorema dasar kalkulus. Penyelesaian hampiran integral tentu dari fungsi diselesaikan dengan bantuan computer menggunakan software Matlab versi 5.3.1

Misalkan $\zeta_{i}=\left(2 x_{i-1}+x_{i}\right) / 3$ dengan $i=1,2, \ldots, n$, sehingga $\xi<<\frac{1}{2}\left(x_{i-1}+x_{i}\right)$ dan misalkan $\Delta x_{1}=\Delta x_{2}=\cdots=\Delta x_{n}$ sechinga $\Delta x_{i}=\frac{b-a}{n}$ dengan $i=1,2, \ldots, n$

\section{Fungsi Polinom}

Perhatikan beberapa contoh berikut

a. Mencari suatu nilai hampiran integral tentu berikut:

$\int_{1}^{4}\left(x^{4}-3\right) d x$

dengan $n=100$, kemudian hasilnya dibandingkan dengan jumlah Riemann dan metode trapesium.

Penyelesaian:

Misalkan $f(x)=x^{4}-3$, maka

$f^{\prime}(x)=f^{\prime}\left(x^{4}-3\right)=f^{\prime}\left(x^{4}\right)-f^{\prime}(3)=f^{\prime}\left(x^{4}\right)-f^{\prime}(3)=4 x^{3}-0=4 x^{3}$

Karena $f(x)$ memiliki turunan pada $[1,4]$, maka hampiran dari $\int_{1}^{4}\left(x^{4}-3\right) d x$ dapat dihitung menggunakan metode garis singgung, dan $f^{\prime}(x)>0$

Nilai eksak berdasarkan Teorema Dasar Kalkulus

$$
\int_{1}^{4} x^{4}-3 d x=\frac{1}{5}\left(x^{5}\right)-\left.3 x\right|_{1} ^{4}=195.6000
$$

- menggunakan (1) dalam metode garis singgung dengan $n=100$ maka $\int_{1}^{4}\left(x^{4}-3\right) d x \approx 195.5874$

- menggunakan jumlah Riemann dengan $n=100$ maka $\int_{1}^{4}\left(x^{4}-3\right) d x \approx 194.3187$

- menggunakan metode trapesium dengan $n=100$ maka $\int_{1}^{4}\left(x^{4}-3\right) d x \approx 195.6189$

Dengan
$r_{\Omega}$ metode garis singgung $=\frac{|195.6000-195.5874|}{|195.6000|}=6.4417 \times 10^{-5}$

$r_{8}$ jumlah Riemann $=\frac{|195.6000-194.3187|}{|195.6000|}=660 \times 10^{-5}$

$r_{\Omega}$ metode trapesium $=\frac{|195.6000-195.6189|}{|195.6000|}=9.6625 \times 10^{-5}$

$r_{\&}$ metode garis singgung $<r_{\ell}$ metode trapesium $<r_{\&}$ jumlah Riemann dan disimpulkan bahwa nilai hampiran dengan metode garis singgung lebih baik dari nilai hampiran jumlah Riemann dan metode trapesium.

b. Mencari suatu nilai hampiran integral tentu berikut:

$$
\int_{-1}^{4}|x| d x
$$

dengan $n=100$, kemudian hasilnya dibandingkan dengan jumlah Riemann dan metode trapesium.

Penyelesaian:

Misalkan $f(x)=|x|$, tetapi $f(x)$ tidak memiliki turunan di $x=0$, maka $f(0)$ dihitung dengan

Limit ini tidak ada karen

$$
f^{\prime}(0)=\lim _{x \rightarrow 0} \frac{f(x)-f(0)}{x-0}=\lim _{x \rightarrow 0} \frac{|x|-|0|}{x}=\lim _{x \rightarrow 0} \frac{|x|}{x}
$$

sedangkan

$$
\lim _{x \rightarrow 0^{+}} \frac{|x|}{x}=\lim _{x \rightarrow 0^{+}} \frac{x}{x}=1
$$

$$
\lim _{x \rightarrow 0^{-}} \frac{|x|}{x}=\lim _{x \rightarrow 0^{-}} \frac{-x}{x}=-1
$$

maka nilai hampiran dari $\int_{-1}^{4}|x| d x$ tidak dapat dihitung dengan metode garis singgung

$$
\begin{aligned}
& \text { c. Fungsi Sinus dan Cosinus } \\
& \text { Mencari suatu nilai hampiran integral tentu berikut: } \\
& \qquad \int_{0}^{\frac{1}{2}} \sin x d x
\end{aligned}
$$

dengan $n=10$, kemudian hasilnya dibandingkan dengan jumlah Riemann dan metode trapesium.

Penyelesaian:

Misalkan $f(x)=\sin x$ maka $f^{\prime}(x)=\cos x$

Karena $f(x)$ memiliki turunan pada $\left[0, \frac{1}{2} \pi\right]$, maka hampiran dari $\int_{0}^{\frac{1}{2}} \pi \sin x d x$ dapat dihitung menggunakan metode garis singgung, dan $f^{\prime}(x)>0$

Nilai eksak

$$
\sin x d x=-\left.\cos x\right|_{0} ^{\frac{1}{2^{\pi}}}=1
$$

- Menggunakan dalam (1) metode garis singgung dengan $n=10$ maka $\int_{0}^{\frac{1}{2} \pi} \sin x d x \approx 1.0014$

- menggunakan jumlah Riemann dengan $n=10$ maka $\int_{0}^{\frac{1}{2}} \sin x d x \approx 0.9745$

- menggunakan metode trapesium dengan $n=10$ maka $\int_{0}^{2 \pi} \sin x d x \approx 0.9979$

dengan

$r_{8}$ metode garis singgung $=\frac{|1-1.0014|}{|1|}=0.0014$

$r_{8}$ jumlah Riemann $=\frac{|1-0.9745|}{|1|}=0.0255$

$r_{R}$ metode trapesium $=\frac{|11-0.9979|}{|1|}=0.0021$

$r_{R}$ metode garis singgung $<r_{z}$ metode trapesium $<r_{\&}$ jumlah Riemann dan disimpulkan bahwa nilai hampiran dengan metode garis singgung lebih baik dari nilai hampiran jumlah Riemann dan metode trapesium. 
d. Fungsi rasional

Mencari suatu nilai hampiran integral tentu berikut:

$$
\int_{2}^{4} \frac{1+x}{x} d x
$$

dengan $n=100$, kemudian hasilnya dibandingkan dengan hampiran jumlah Riemann dan metode trapesium.

Penyelesaian:

Misalkan $f(x)=\frac{1+x}{x}$, maka $f^{\prime}(x)=\frac{1}{x}-\frac{1+x}{x^{2}}$

Karena $f(x)$ memiliki turunan pada $[2,4]$, maka hampiran dari $\int_{2}^{4} \frac{1+x}{x} d x$ dapat dihitung menggunakan metode garis singgung, dan $f^{\prime}(x)<0$

Nilai eksak

$$
\int_{2}^{4} \frac{1+x}{x} d x=\ln (x)+\left.x\right|_{2} ^{4}=2.6931
$$

- menggunakan dalam (1) metode garis singgung dengan $n=100$ maka $\int_{2}^{4} \frac{1+x}{x} d x \approx 2.6931$

- menggunakan jumlah Riemann dengan $n=100$ maka

$\int_{2}^{4} \frac{1+x}{x} d x \approx 2.6940$

- menggunakan metode trapesium dengan $n=100$ maka $\int_{2}^{4} \frac{1+x}{x} d x \approx 2.6932$

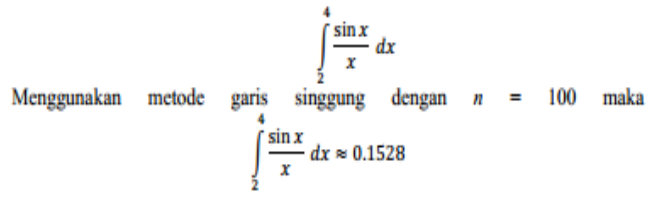

